\title{
Non-Monte Carlo Simulation and Sensitivity of Linear(ized) Analog Circuits under Parameter Variations*
}

\author{
C.-J. Richard Shi and Michael W. Tian \\ University of Iowa, ECE Department \\ Iowa City, Iowa 52242, USA
}

\begin{abstract}
In this paper, a non-Monte Carlo approach is presented for the simulation and sensitivity analysis of linear(ized) analog circuits under parameter variations. With uncertain parameters represented as intervals, we present a formulation of circuit equations as a set of interval linear equations. An elegant and efficient algorithm is developed based on some interval-mathematic results derived in this paper. In contrast to Monte Carlo simulation, the proposed approach is rapid-with the computational cost comparable to one nominal circuit simulation-and correct-the computed bounds always contain, and are usually close to, the actual bounds. Further, sensitivity under parameter variations can be computed from the response bounds with minor computation cost. The algorithms have been implemented into SPICE3F5. Experimental results are promising.
\end{abstract}

\section{INTRODUCTION}

Recent developments in microelectronic manufacturing technologies and system design methodologies have created a new set of challenges to circuit simulation. This has been exemplified in three aspects: First, the complexity of microelectronic systems being designed is increasing constantly in the number of components being integrated. As a result, simulation and verification are taking enormous amount of computation time. Second, the types of components found in a chip (system-on-achip) are becoming radically diversified, e.g., electronic devices, as well as optical devices, micro-electro-mechanical (MEMS) devices. As a consequence, circuit parameters are more subject to manufacturing process variations. For example, embedded passive components (resistors and capacitors) in mixed-signal multi-chip modules have a typical variation of $\pm 10 \%$ [2]. Parameter uncertainty arises also in multi-abstraction/mixed-mode simulation. Higher level abstraction usually represents some uncertainty to lower level simulation. For example, unknown states in

*This work is sponsored by U.S. Defense Advanced Research Projects Agency (DARPA) under grant number F33615-96-1-5601 from the U.S. Air Force, Wright Laboratory, Manufacturing Technology Directorate, and by the National Science Foundation under grant CDA 96-01503. 
digital portion give rise to parameter uncertainty in analog portion. Finally, the timeto-market pressure and life-cycle-costs motivate more use of simulation as a tool not only in the design process, but also in the test development stage. In this paper, we consider how to simulate efficiently electronic circuits under parameter variations.

Simulation under parameter variations was studied early in the area of design for manufacturing. Since only extreme conditions are considered, it is known as worstcase tolerance analysis $[8,11,12]$. Existing approaches can be classified into three categories: vertex enumeration, sensitivity-based estimation, and Monte Carlo simulation. Vertex enumeration is to simulate all the possible extreme parameter cases. However, extreme values of an output response may not necessary occur at the vertices of parameter space. Even if the extreme value occurs at the vertices, as this is the case of resistive networks [1], finding such a extreme value is NP-complete, as proved by Huang and Bryant in the context of switch-level simulation [7].

The sensitivity-based approach is to calculate the worst-case response via sensitivity estimation. Suppose that $f$ is the function, and $x_{i}$ is a statistical parameter. Then the upper and lower bounds of $f$ under parameter variations can be estimated as as

$f_{0}-\sum\left|\frac{\partial f}{\partial x_{i}}\left\|\delta x_{i}\left|, \quad f_{0}+\sum\right| \frac{\partial f}{\partial x_{i}}\right\| \delta x_{i}\right|$

where $f_{0}$ is the nominal value of $f$. This method is not computationally expensive. However, it is not predictable whether the results underestimate or overestimate the actual bounds.

With the Monte Carlo technique, simulation is repeated for random combinations of values chosen from within the range of each parameter. Unfortunately, determining accurate bounds on the behavior of a circuit requires a large number of simulations to be effective. Therefore, its use is limited to small circuits that have a few statistical parameters. In addition, the Monte Carlo method always underestimates the response bounds.

In this paper, we present a rapid, correct and conservative approach to frequencydomain simulation and sensitivity analysis of linear(ized) analog circuits and systems under parameter tolerances. A large class of circuits and systems widely used in video and image processing, digital signal processing, control, communications, and many other applications falls into this category. The proposed method is based on interval mathematics. We note that the use of interval analysis to circuit simulation was not a new idea. However, as pointed out by Zukowski [14], "the field of interval arithmetic, which has often addressed very general problems, has developed a poor reputation". Previous attempts have not produced satisfactory results $[8,11]$. Our approach is based on two techniques described in this paper. First, a novel formulation of circuit equations, called generalized nodal analysis, is developed. Second, we derive a theoretical characterization of interval linear systems. Based on the theory, an elegant and efficient algorithm is developed. Our work is inspired by the recent work of Hansen [4].

This paper details our theory and methods, and presents a prototype analog circuit simulator utilizing the proposed techniques. An interval-based theoretical framework 
for handling parameter variations is introduced in Section 2. Under this framework, circuit equations are formulated by generalized modified nodal analysis as a set of interval linear equations in Section 3. A theory is developed in Section 4, which leads to an elegant and efficient algorithm described in Section 5 . Sensitivity under process variations are obtained as a by-product with virtually no extra computation cost, as shown in Section 6. Implementation and experimental results are described in Section 7. Section 8 concludes the paper.

\section{NOTATIONS FROM INTERVAL MATHEMATICS}

In this section we present some concepts and notations for later use from interval mathematics.

Let $p \in \mathcal{R}$ be a real number whose value may not be precisely known. Instead, we are often given a range and $p$ is uncertain within this range. This can be represented by an interval number $p^{I}$, with lower (left) bound $p^{L}$ and upper (right) bound $p^{R}$, denoted by $p^{I}=\left[p^{L}, p^{R}\right]$. The midpoint mid $\left(p^{I}\right)$ of an interval $p^{I}$ is defined as $\operatorname{mid}\left(p^{I}\right)=\frac{1}{2}\left(p^{R}+p^{L}\right)$, and the radius $\operatorname{rad}\left(p^{I}\right)$ of $p^{I}$ is defined as $\operatorname{rad}\left(p^{I}\right)=$ $\frac{1}{2}\left(p^{R}-p^{L}\right)$.

An interval vector $\mathbf{x}^{I}$ is a vector whose elements are interval numbers, and we write an interval vector as $\mathbf{x}^{I}=\left[\mathbf{x}^{L}, \mathbf{x}^{R}\right]$. An interval matrix $\mathbf{A}^{I}$ is a matrix whose elements are interval numbers. We write an interval matrix as $\mathbf{A}^{I}=\left[\mathbf{A}^{L}, \mathbf{A}^{R}\right]$. The midpoint and radius of an interval vector and matrix are defined similar to that of an interval number.

We say an interval number $p^{I}$ contains $q^{I}$, denoted as $q^{I} \subseteq p^{I}$, iff $p^{L} \leq q^{L} \leq$ $q^{R} \leq p^{R}$. An interval vector $\mathbf{x}^{I}$ or matrix $\mathbf{A}^{I}$ contains interval vector $\mathbf{y}^{I}$ or matrix $\mathbf{B}^{I}$ is every element of $\mathbf{x}^{I}$ or $\mathbf{A}^{I}$ contains the corresponding element of $\mathbf{y}^{I}$ or $\mathbf{B}^{I}$.

An interval linear equation, $\mathbf{A}^{I} \mathbf{x}=\mathbf{b}^{I}$, is defined as the family set of linear equations:

$\left\{\mathbf{A x}=\mathbf{b} \mid \mathbf{A} \in \mathbf{A}^{I}\right.$ and $\left.\mathbf{b} \in \mathbf{b}^{I}\right\}$.

We are interested in the solution set, given by:

$S=\left\{\mathbf{x}=\mathbf{A}^{-1} \mathbf{b} \mid \mathbf{A} \in \mathbf{A}^{I}\right.$ and $\left.\mathbf{b} \in \mathbf{b}^{I}\right\}$.

In general, the solution set $S$ is a nonempty convex subset in $\mathcal{R}^{n}$. The solution hull is defined as the tightest interval enclosing $S$, represented as:

$\left(\mathbf{A}^{I}\right)^{H} \mathbf{b}^{I}=\square\left\{\mathbf{x}=\mathbf{A}^{-1} \mathbf{b} \mid \mathbf{A} \in \mathbf{A}^{I}\right.$ and $\left.\mathbf{b} \in \mathbf{b}^{I}\right\}$.

Here $\square$ is to take the hull of a non-empty set, and $\left(\mathbf{A}^{I}\right)^{H} \mathbf{b}^{I}$ denotes the result of mapping $\left(\mathbf{A}^{I}\right)^{H}$ applied to $\mathbf{b}^{I}$. The projection of a solution hull into any particular dimension is an interval. With a slightly abuse of notation, we use $x^{I}$ to denote the solution hull, and refer to $x_{i}^{R}$ and $x_{i}^{L}$ as solution bounds. 


\section{GENERALIZED MNA FORMULATION OF CIRCUIT EQUATIONS}

One well-known peculiar factor of interval mathematics is that the order of operations affects the solution quality significantly. In this section, we present a specific formulation of circuit equations such that its later solution yields tight bounds.

Consider a linear time-invariant circuit, where some circuit parameters are uncertain, and represented by interval numbers. The Modified Nodal Analysis (MNA) method [6] is adopted with slight modification to formulate the circuit equations. Basically, for all the components which do not have interval parameters, the rules of MNA are followed. For every component with an interval parameter, an additional variable (branch current) and an additional equation (branch equation) are introduced. This is called generalized MNA formulation, or simply Generalized Nodal Analysis) (GNA). In general, the GNA formulation results in a set of interval complex linear equations, represented as:

$\mathbf{T}^{I} \mathbf{x}^{I}=\mathbf{w}^{I}$

Interval complex equations in Eq. (1) can be rewritten as a set of interval real equations as follows:

$$
\left(\begin{array}{cc}
\mathbf{T}_{\mathcal{R}}^{I} & -\mathbf{T}_{\mathcal{I}}^{I} \\
\mathbf{T}_{\mathcal{I}}^{I} & \mathbf{T}_{\mathcal{R}}^{I}
\end{array}\right)\left(\begin{array}{c}
\mathbf{x}_{\mathcal{R}}^{I} \\
\mathbf{x}_{\mathcal{I}}^{I}
\end{array}\right)=\left(\begin{array}{c}
\mathbf{w}_{\mathcal{R}}^{I} \\
\mathbf{w}_{\mathcal{I}}^{I}
\end{array}\right)
$$

where subscripts $\mathcal{R}$ and $\mathcal{I}$ denote, respectively, the real part and the imaginary part of a complex matrix, a complex vector, or a complex number. Therefore, under our formulation, frequency-domain circuit simulation under parameter variations amounts to solving Eq. (2)-a set of interval linear equations-for a given set of frequency points.

\section{PRECONDITIONING OF INTERVAL LINEAR SYSTEMS AND ITS CHARACTERIZATION}

In this section, we present a formalization of some original ideas described by Hansen [4] for solving the system of interval linear equations in the form of

$\mathbf{A}^{I} \mathbf{x}=\mathbf{b}^{I}$

Let $\mathbf{C}$ be the midpoint of interval matrix $\mathbf{A}^{I}$, i.e., $\mathbf{C}=\frac{1}{2}\left(\mathbf{A}^{L}+\mathbf{A}^{R}\right)$. Pre-multiplying Eq. (3) with $\mathbf{C}^{-1}$ transforms the original system into a new system:

$\mathbf{M}^{I} \mathbf{x}=\mathbf{r}^{I}$ 
where $\mathbf{M}^{I}=\mathbf{C}^{-1} \mathbf{A}^{I}$ and $\mathbf{r}^{I}=\mathbf{C}^{-1} \mathbf{b}^{I}$. We refer to this transformation as preconditioning, and call Eq. (4) the preconditioned system.

An assumption used in this paper is as follow:

Assumption 1 The preconditioned interval matrix $\mathbf{M}^{I}$ is regular, i.e., every component matrix $\mathbf{M}, \mathbf{M} \in \mathbf{M}^{I}$, is nonsingular.

The original matrix A is said to be strongly regular if Assumption 1 is satisfied.

Proposition 1 The solution hull of the preconditioned system contains that of the original system, i.e., $\left(\mathbf{A}^{I}\right)^{H} \mathbf{b}^{I} \subseteq\left(\mathbf{M}^{I}\right)^{H} \mathbf{r}^{I}$.

Proposition 2 The solution hull of the preconditioned system contains the right hand side vector, i.e., $\mathbf{r}^{I} \subseteq\left(\mathbf{M}^{I}\right)^{H} \mathbf{r}^{I}$.

Proposition 3 The preconditioned matrix $\mathbf{M}^{I}$ is centered around the identity matrix I, i.e., $M_{i j}^{R}=-M_{i j}^{L}>0$, for $i \neq j$ and $M_{i i}^{R}+M_{i i}^{L}=2$.

Since $\mathbf{M}^{I}$ does not contain a singular matrix (Assumption 1), and is centered around the identity matrix (Proposition 1), it follows:

Proposition 4 The preconditioned matrix $\mathbf{M}^{I}$ satisfies the following properties:

$0<M_{i i}^{L} \leq 1 \quad 1 \leq M_{i i}^{R}<2$

Theorem 1 The solution set of the preconditioned system (4) is defined by the following set of inequalities:

$\mathbf{M}^{L}|\mathbf{x}| \leq \mathbf{Y m i d}\left(\mathbf{r}^{I}\right)+\operatorname{rad}\left(\mathbf{r}^{I}\right)$
$\mathbf{M}^{R}|\mathbf{x}| \geq \mathbf{Y m i d}\left(\mathbf{r}^{I}\right)-\operatorname{rad}\left(\mathbf{r}^{I}\right)$

where $\mathbf{Y}$ is a diagonal matrix such that

$Y_{i j}=\left\{\begin{array}{lr}0 & \text { if } i \neq j \\ 1 & \text { if } i=j \text { and } x_{i} \geq 0 \\ -1 & \text { if } i=j \text { and } x_{i} \leq 0\end{array}\right.$

Theorem 2 For the preconditioned system (4), the maximum and minimum non-zero values of $\left|x_{i}\right|$ are determined respectively by the following two sets of equations:

$$
\begin{aligned}
\mathbf{M}^{L}|\mathbf{x}| & =\left|\operatorname{mid}\left(\mathbf{r}^{\mathbf{I}}\right)\right|+\operatorname{rad}\left(\mathbf{r}^{I}\right) \\
\left(\mathbf{M}^{L}-2 \mathbf{e}_{i} \mathbf{e}_{i}^{T}\right)|\mathbf{x}| & =\left(\mathbf{I}-2 \mathbf{e}_{i} \mathbf{e}_{i}^{T}\right)\left|\operatorname{mid}\left(\mathbf{r}^{\mathbf{I}}\right)\right|+\operatorname{rad}\left(\mathbf{r}^{I}\right)
\end{aligned}
$$


where $\mathbf{e}_{i}$ is the ith unit vector whose ith component is 1 and all other components are 0 , and superscript $T$ denotes the transpose.

\section{AN ALGORITHM FOR SOLVING PRECONDITIONED INTERVAL LINEAR SYSTEMS}

In this section, we describe an algorithm for solving a set of preconditioned interval linear equations, based on the theory developed in the last section.

For notational convenience, let us define a vector $s \in \mathcal{R}^{n}$ such that

$s_{i}=\left|\operatorname{mid}\left(r_{i}^{I}\right)\right|+\operatorname{rad}\left(r_{i}^{I}\right)$,

and $\mathbf{t}^{(\mathbf{i})} \in \mathcal{R}^{n}$ such that

$t_{j}^{(i)}=\left\{\begin{array}{cc}\operatorname{rad}\left(r_{j}^{I}\right)-\left|\operatorname{mid}\left(r_{j}^{I}\right)\right| & \text { if } j=i \\ s_{j} & \text { otherwise }\end{array}\right.$

Let us define $\mathbf{P}=\left(\mathbf{M}^{L}\right)^{-1}$, and

$$
\begin{aligned}
f_{i} & =\sum_{j=1}^{n} P_{i j} s_{j}, \\
g_{i} & =\sum_{j=1}^{n} P_{i j} t_{j}^{(i)} .
\end{aligned}
$$

Then from Theorem 2, the maximum value of $\left|x_{i}\right|$ equals $f_{i}$. Now we consider how to compute the minimum non-zero value of $\left|x_{i}\right|$. According to Householder's inverse matrix formula [5], we have:

$$
\left(\mathbf{M}^{L}-2 \mathbf{e}_{i} \mathbf{e}_{i}^{T}\right)^{-1}=\mathbf{P}-2 c_{i} \mathbf{P} \mathbf{e}_{i} \mathbf{e}_{i}^{T} \mathbf{P},
$$

where $c_{i}=1 /\left(2 P_{i i}-1\right)$. Hence, the minimum value of $\left|x_{i}\right|$ is equal to

$$
\begin{aligned}
\mathbf{e}_{i}^{T}\left(\mathbf{M}^{L}-2 \mathbf{e}_{i} \mathbf{e}_{i}^{T}\right)^{-1} \mathbf{t}^{(i)} & =\mathbf{e}_{i}^{T}\left(\mathbf{M}^{L}-2 \mathbf{e}_{i} \mathbf{e}_{i}^{T}\right)^{-1} \mathbf{t}^{(i)} \\
& =\mathbf{e}_{i}^{T}\left(I-2 c_{i} \mathbf{P} \mathbf{e}_{i} \mathbf{e}_{i}^{T}\right) \mathbf{P t}^{(i)} \\
& =-c_{i} \sum_{j=1}^{n} P_{i j} t_{j}^{(i)} \\
& =-c_{i} g_{i} .
\end{aligned}
$$

Now we are ready to present the complete algorithm for computing the solution hull of the preconditioned system. The algorithm is summarized in pseudo-code in Fig. 1. 


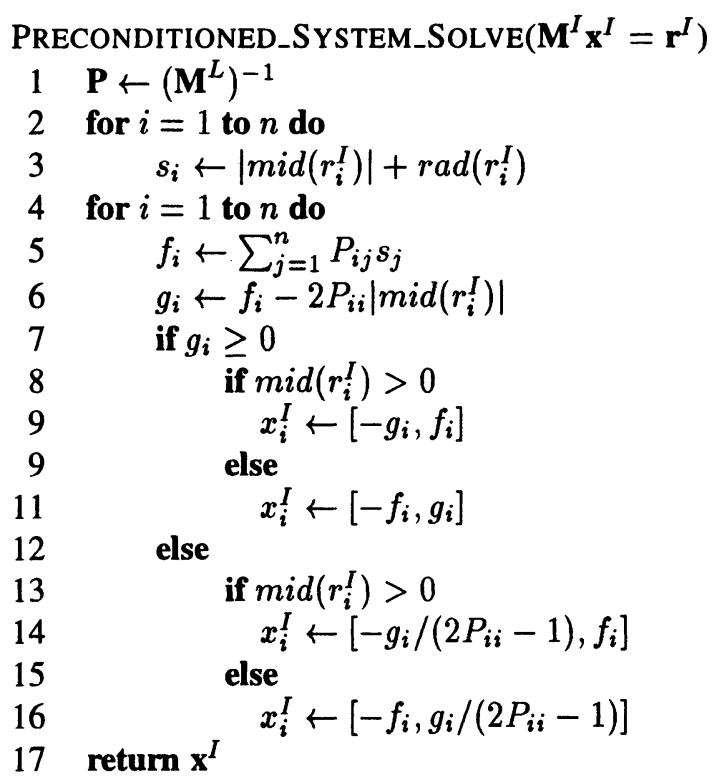

Figure 1 An algorithm for solving preconditioned interval linear systems.

Theorem 3 Algorithm PRECONDITIONED_SYSTEM_SOLVE computes the exact solution hull of the preconditioned system (4) with time complexity $O\left(n^{3}\right)$.

\section{SENSITIVITY COMPUTATION UNDER PARAMETER VARIATIONS}

Sensitivity is an important tool in many contexts of circuit design and test $[10,12]$. We describe here a method to compute sensitivity under parameter variations with minor computational cost.

Consider the circuit equation set shown in Eq. (1). In order to evaluate the sensitivities of all components of the vector $\mathbf{x}^{I}$ to a single parameter $p_{i}^{I}$, we differentiate Eq. (1) with respect to $p_{i}^{I}$ to obtain:

$\frac{\partial \mathbf{T}^{I}}{\partial p_{i}^{I}} \mathbf{x}^{I}+\mathbf{T}^{I} \frac{\partial \mathbf{x}^{I}}{\partial p_{i}^{I}}=\frac{\partial \mathbf{w}^{I}}{\partial p_{i}^{I}}$

Because vector $\mathbf{w}^{I}$ does not depend on $p_{i}^{I}$, the equation set above can be rewritten as $\mathbf{T}^{I} \frac{\partial \mathbf{x}^{I}}{\partial p_{i}^{I}}=-\frac{\partial \mathbf{T}^{I}}{\partial p_{i}^{I}} \mathbf{x}^{I}$

Note that in the GNA formulation, every uncertain parameter appears only in one 
matrix entry. Suppose matrix entry $t_{p q}^{I}$ is for component $p_{i}^{I}$, then we have:

$\mathbf{T}^{I} \frac{\partial \mathbf{x}^{I}}{\partial p_{i}^{I}}=-\frac{\partial t_{p q}^{I}}{\partial p_{i}^{I}} \frac{\partial \mathbf{T}^{I}}{\partial t_{p q}^{I}} \mathbf{x}^{I}$

Note that the complex equation set Eq. (1) is converted to a real equation set Eq. (2) for solution. Each matrix entry in Eq. (1) leads to two matrix entries in Eq. (2). Suppose that for $t_{p q}$, the two corresponding matrix entries in Eq. (2) are $a_{k l}$ and $a_{m n}$, then the above equation set can be transferred to a real equation set as

$$
\mathbf{A}^{I} \frac{\partial \mathbf{x}^{I}}{\partial p_{i}^{I}}=-\frac{\partial t_{p q}^{I}}{\partial p_{i}^{I}} \hat{\mathbf{b}}
$$

where $\partial t_{p q}^{I} / \partial p_{i}^{I}$ is very easy to obtain from our GNA formulation method and:

$\hat{b}_{i}=\left\{\begin{array}{cc}x_{l}^{I} & \text { if } i=k \text { and } p_{i}^{I} \text { is real number } \\ x_{n}^{I} & \text { if } i=m \text { and } p_{i}^{I} \text { is real number } \\ -x_{l}^{I} & \text { if } i=k \text { and } p_{i}^{I} \text { is imaginary number } \\ -x_{n}^{I} & \text { if } i=m \text { and } p_{i}^{I} \text { is imaginary number } \\ 0 & \text { otherwise. }\end{array}\right.$

Now sensitivity computation under parameter variations amounts to solving interval linear equation set Eq. (11). Note that Eq. (11) has the same form as Eq. (3): only the right hand side is different. Since Eq. (3) has been solved by algorithm PRECONDITIONED_SYSTEM_SOlVE in Section 5, the vector $\mathbf{x}^{I}$ is known. Thus the right hand side vector can be easily generated. Since the inverse matrix $\mathbf{P}$ is already available, solution of Eq. (11) involves executing Lines 2-16 in the algorithm, which takes time linear in the number of non-zero entries in $\mathbf{P}$.

\section{EXPERIMENTAL RESULTS}

The proposed algorithms have been implemented into a computer program SIVA$A C$ using the SPICE3F5 sparse matrix package. A number of experiments have been conducted mainly to test the quality of computed interval bounds and validity of sensitivity computation. In this section, we describe two examples. All CPU data are collected on a SPARC-Ultra 1 workstation.

\subsection{A Two-Amplifier Active Network}

An active network shown in Fig. 2 is used to compare the quality of response bounds produced by the proposed interval method with those by the existing Monte Carlo 
method, ad well as the sensitivity-based method. The nominal parameter values are given as

$R_{1}=1, R_{2}=1, C_{1}=1, C_{2}=1, E_{1}=1.3784, E_{2}=1.3784$.

All the parameters are assigned $\pm 1 \%$ statistical variations.

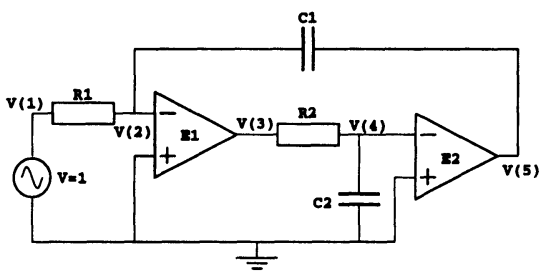

Figure 2 Circuit schematic of a two-amplifier active network.

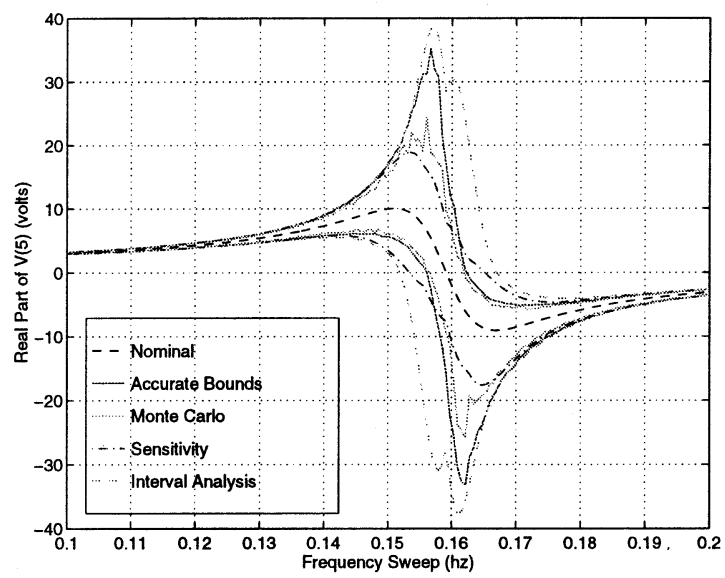

Figure 3 Real part of $V(5)$.

Figure 3 plots the results of the real part of $V(5)$ computed by various methods. We observe that, the Monte Carlo simulation ( $10^{4}$ trials) always under-estimates the accurate bounds (approximated by $10^{6}$ trials Monte Carlo simulation). Our interval method always over-estimates the accurate bounds. The nominal-sensitivity-based method may over-estimates the actual bounds in some frequency ranges (e.g., lower bounds between $0.145 h z-0.155 h z$ ), and may under-estimate that in some other frequency ranges (e.g., upper bounds between $0.155 h z-0.16 h z$ ). Our interval method is superior to others, since the computed bands always contain the actual bands, and further the differences are usually small. 


\section{2 $\mu$ A 741 Operational Amplifier}

We further test our program on the the more complex $\mu \mathrm{A} 741$ operational amplifier, shown in Fig. 4. The nominal values of circuit parameters are

$$
\begin{array}{r}
R_{1}=1 k, R_{2}=50 k, R_{3}=1 k, R_{4}=3 k, R_{5}=39 k, R_{6}=50 \\
R_{7}=25, R_{8}=100, R_{9}=50 k, R_{10}=40 k, R_{11}=50 k, \text { COMP }=30 p f .
\end{array}
$$

All the parameters are assigned $\pm 5 \%$ statistical variations.

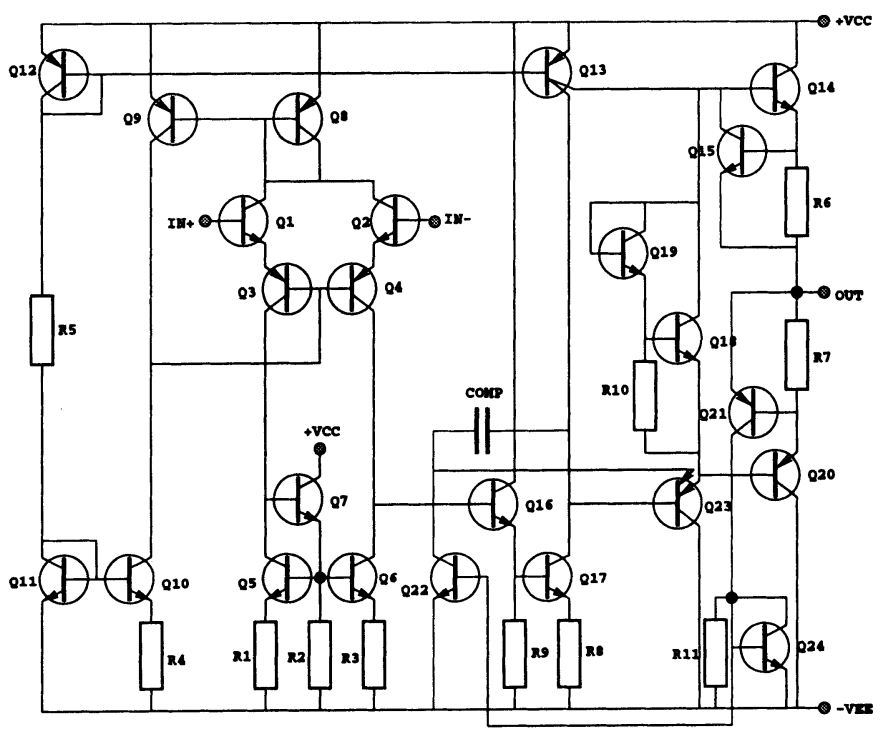

Figure $4 \mu \mathrm{A} 741$ operational amplifier circuit.

Figures 5(a) and 5(b) plot the output bands of $V(O U T)$ under parameter variations computed by our method, as well as by Monte Carlo. For 80 frequency points, SIVA-AC took 68.2 second CPU time, Monte Carlo (10000 trails) took 12000 seconds. Figures 6(a) and 6(b) show the sensitivity of $V(O U T)$ with respect to Ccomp under parameter variations computed by our method, as well as the nominal sensitivity computed by SPICE3F5. We observe that the computed sensitivity bands contain the curves of nominal sensitivity, and further, the shapes of sensitivity bands match those of the nominal sensitivity. For 80 frequency points, SIVA-AC took 22.7 seconds, while SPICE nominal simulation took 24.5 seconds.

\section{CONCLUDING REMARKS}

An interval-based approach was presented for frequency-domain simulation and sensitivity analysis of linear(ized) analog integrated circuits and systems under param- 


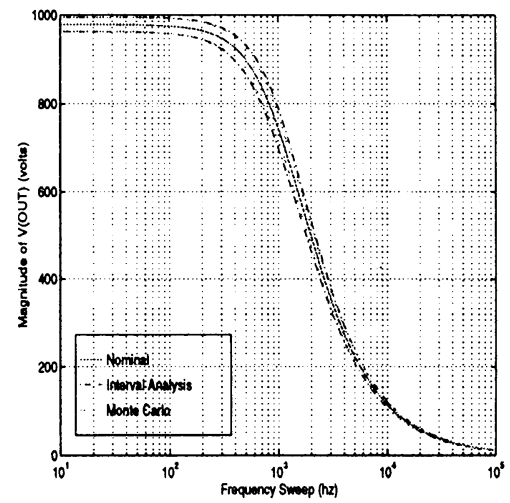

(a) magnitude of $V(O U T)$

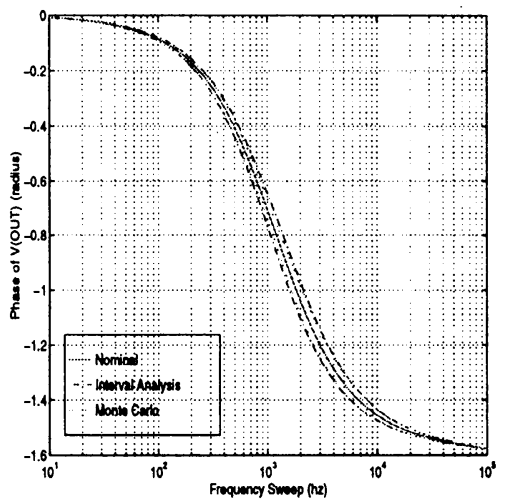

(b) phase of $V(O U T)$

Figure 5 Simulation results under parameter variations.

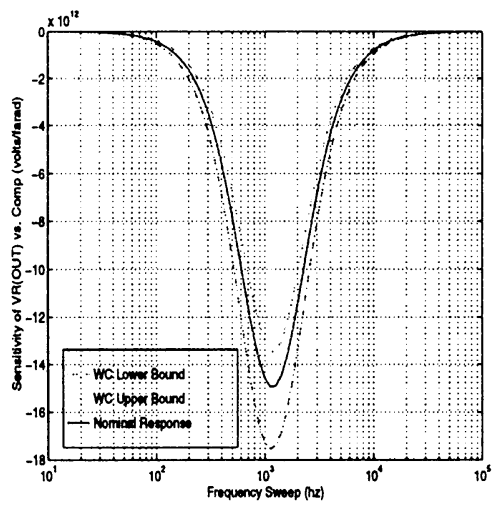

(a) real part of $V(O U T)$

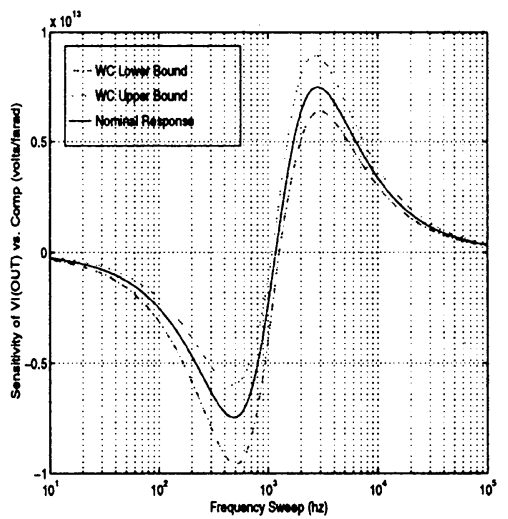

(b) imaginary part of $V(O U T)$

Figure 6 Sensitivity computation results under parameter variations.

eter variations. Experimental results have demonstrated that the proposed method is extremely fast, and usually produces the bounds very close to actual bounds. We attribute this to both a careful formulation of circuit equations and an elegant algorithm developed on a series of theoretical characterization derived in this paper.

We note that what we addressed in this paper is circuit simulation under parameter variations. An application of this technique to analog fault simulation appears in [13]. The statistical variations are completely ignored in our framework. As a consequence, this method is not applicable to the situations where statistical distribution is significant such as yield estimation [3]. 


\section{REFERENCES}

[1] R. K. Brayton, A. J. Hoffman, and T. R. Scott, "A theorem on inverse of convex sets of real matrices with application to the worst case DC problem", IEEE Trans. Circuits and Systems, vol. 24, no. 8, pp. 409-415, Aug. 1977.

[2] Proceedings of Electronic Interconnect and Packaging Program Review, U.S. Defense Advanced Research Projects Agency, Oct. 30, 1996.

[3] S. W. Director, W. Maly and A. J. Strojwas, VLSI Design for Manufacturing: Yield Enhancement, Kluwer Academic Publishers, 1990.

[4] E. R. Hansen, "Bounding the solution of interval linear equations", SIAM J. Numerical Analysis, vol. 29, no. 5, pp. 1493-1503, Oct. 1992.

[5] A. S. Householder, "A survey of some closed methods for inverting matrices," SIAM J. Appl. Math., vol. 5, pp. 155-169, 1957.

[6] C. W. Ho, A. E. Ruehli and P. A. Brennan, "The modified nodal approach to network analysis", IEEE Trans. Circuits and Systems, vol. 22, pp. 504-509, June 1975.

[7] L. P. Huang and R. E. Bryant, "Intractability in linear switch-level simulation", IEEE Trans. Computer-Aided Design, vol. 12, pp. 829-836, June 1993.

[8] L. V. Kolev, V. M. Mladenov, and S. S. Vladov, "Interval mathematics algorithms for tolerance analysis", IEEE Trans. Circuits and Systems, vol. 35, no. 8, pp. 967-974, August 1988.

[9] A. Neumaier, Interval Methods for Systems of Equations, Cambridge University Press, 1990.

[10] A. Pahwa and R. A. Rohrer, "Band-faults: Efficient approximations to fault bands for the simulation before fault diagnosis of linear circuits," IEEE Trans. Circuits and Systems, vol. 29, no. 2, pp. 81-88, Feb. 1982.

[11] S. Skelboe, "True worst-case analysis of linear electrical circuits by interval arithmetic", IEEE Trans. Circuits and Systems, pp. 874-879, vol.-26, Oct. 1979.

[12] R. Spence and R. S. Soin, Tolerance Design of Electronic Circuits, AddisonWesley Publishing Company, pp. 27-55, 1988.

[13] Michael W. Tian and C.-J. Richard Shi, "Rapid analog fault simulation under parameter tolerances", in Proc. IEEE/ACM Design Automation Conference, June 1997.

[14] C. A. Zukowski, The Bounding Approach to VLSI Circuit Simulation, Kluwer Academic Publishers, 1986. 